\title{
Anxiety Associated Increased CpG Methylation in the Promoter of Asb I: A Translational Approach Evidenced by Epidemiological and Clinical Studies and a Murine Model
}

\begin{abstract}
Rebecca T Emeny',2, Jens Baumert', Anthony S Zannas ${ }^{3,4}$, Sonja Kunze ${ }^{5}$, Simone Wahl ${ }^{5}$, Stella lurato ${ }^{3}$, Janine Arloth ${ }^{3,6}$, Angelika Erhardt ${ }^{3}$, Georgia Balsevich ${ }^{7}$, Mathias V Schmidt ${ }^{7}$, Peter Weber ${ }^{3}$, Anja Kretschmer ${ }^{5}$, Liliane Pfeiffer ${ }^{5}$, Johannes Kruse ${ }^{8}$, Konstantin Strauch ${ }^{9,10}$, Michael Roden ${ }^{11,12,13}$, Christian Herder ${ }^{1,12}$, Wolfgang Koenig ${ }^{14}$, Christian Gieger ${ }^{5}$, Melanie Waldenberger ${ }^{5}$, Annette Peters', Elisabeth B Binder*,3,15 and Karl-Heinz Ladwig, ${ }^{*, 1,16}$

Institute of Epidemiology II, Helmholtz Zentrum München - German Research Centre for Environmental Health, Neuherberg, Germany;

${ }^{2}$ The Dartmouth Institute for Health Policy and Clinical Practice, Geisel School of Medicine at Dartmouth, Lebanon, NH, USA; ${ }^{3}$ Department of Translational Research in Psychiatry, Max Planck Institute of Psychiatry, Munich, Germany; ${ }^{4}$ Department of Psychiatry and Behavioral Sciences, Duke University Medical Center, Durham, NC, USA; ${ }^{5}$ Research Unit of Molecular Epidemiology and Institute of Epidemiology II, Helmholtz Zentrum München, Neuherberg, Germany; ${ }^{6}$ Institute of Computational Biology, Helmholtz Zentrum München-German Research Center for Environmental Health, Neuherberg, Germany; 'Department of Stress Neurobiology and Neurogenetics, Max Planck Institute of Psychiatry, Munich, Germany; ${ }^{8}$ Department of Psychosomatic Medicine and Psychotherapy Justus-Liebig-Universität Gießen, Gießen, Hesse, Germany; ${ }^{9}$ Institute of Genetic Epidemiology, Helmholtz Zentrum München—German Research Center for Environmental Health, Neuherberg, Germany; ${ }^{10}$ Institute of Medical Informatics, Biometry and Epidemiology, Chair of Genetic Epidemiology, Ludwig-Maximilians-Universität, Munich, Germany; ' 'Institute for Clinical Diabetology, German Diabetes Center, Leibniz Center for Diabetes Research at Heinrich Heine University Düsseldorf, Düsseldorf, Germany; ${ }^{12}$ German Center for Diabetes Research (DZD), München-Neuherberg, Germany; ${ }^{3}$ Department of Endocrinology and Diabetology, Medical Faculty, University Hospital Düsseldorf, Düsseldorf, Germany; ${ }^{14}$ Department of Internal Medicine II-Cardiology, University of Ulm Medical Center, Ulm, Germany; ${ }^{15}$ Department of Psychiatry and Behavioral Sciences, Emory University School of Medicine, Atlanta, GA, USA; ${ }^{16}$ Klinik und Poliklinik für Psychosomatische Medizin und Psychotherapie des Klinikums Rechts der Isar der TUM, Munich, Germany
\end{abstract}

Epigenetic regulation in anxiety is suggested, but evidence from large studies is needed. We conducted an epigenome-wide association study (EWAS) on anxiety in a population-based cohort and validated our finding in a clinical cohort as well as a murine model. In the KORA cohort, participants ( $n=1522$, age 32-72 years) were administered the Generalized Anxiety Disorder (GAD-7) instrument, whole blood DNA methylation was measured (Illumina 450K BeadChip), and circulating levels of hs-CRP and IL-I8 were assessed in the association between anxiety and methylation. DNA methylation was measured using the same instrument in a study of patients with anxiety disorders recruited at the Max Planck Institute of Psychiatry (MPIP, I3I non-medicated cases and I69 controls). To expand our mechanistic understanding, these findings were reverse translated in a mouse model of acute social defeat stress. In the KORA study, participants were classified according to mild, moderate, or severe levels of anxiety $(29.4 \% / 6.0 \% / 1.5 \%$, respectively). Severe anxiety was associated with $48.5 \%$ increased methylation at a single CpG site (cgl270 I57I) located in the promoter of the gene encoding Asb I $(\beta$-coefficient $=0.56$ standard error $(\mathrm{SE})=0.10, p$ (Bonferroni) $=0.005)$, a protein hypothetically involved in regulation of cytokine signaling. An interaction between IL- I 8 and severe anxiety with methylation of this CpG cite showed a tendency towards significance in the total population $(p=0.083)$ and a significant interaction among women $(p=0.014)$. Methylation of the same CpG was positively associated with Panic and Agoraphobia scale (PAS) scores $(\beta=0.005, \mathrm{SE}=0.002, p=0.021, n=|3|$ ) among cases in the MPIP study. In a murine model of acute social defeat stress, Asb I gene expression was significantly upregulated in a tissue-specific manner $(p=0.006)$, which correlated with upregulation of the neuroimmunomodulating cytokine interleukin I beta. Our findings suggest epigenetic regulation of the stress-responsive Asb / gene in anxiety-related phenotypes. Further studies are necessary to elucidate the causal direction of this association and the potential role of Asb I-mediated immune dysregulation in anxiety disorders.

Neuropsychopharmacology (2018) 43, 342-353; doi:I0.1038/npp.2017.102; published online 21 June 2017

\footnotetext{
*Correspondence: Dr EB Binder, Department of Translational Research in Psychiatry, Max Planck Institute of Psychiatry, Kraepelinstr. 2- I0, Munich 80804, Germany, Tel: +49 89 30622586, Fax: +49 89 3062247I or Dr K-H Ladwig, Institute of Epidemiology II, Mental Health Research Unit Helmholtz Zentrum München German Research Center for Environmental Health (GmbH) Ingolstädter Landstr. I, Neuherberg 85764, Germany, Tel: +4989 31873623, Fax: +498931873364, E-mail: binder@psych.mpg.de or ladwig@helmholtz-muenchen.de Received 18 May 2016; revised 4 April 2017; accepted 3 May 2017; accepted article preview online 25 May 2017
} 


\section{INTRODUCTION}

Anxiety encompasses overwhelming feelings of worry and fear. Fear responsiveness is an evolutionarily hard-wired reaction to a perceived threat that facilitates life-dependent, appropriate cognitive and behavioral responses. Pathology is considered when an individual experiences unfocused worry and an excessive and uncontrollable overreaction to a stimulus that is subjectively perceived as menacing (Tyrer and Baldwin, 2006). The spectrum of anxiety disorders includes distress- (generalized anxiety disorder) and fear-disorder clusters (ie, panic disorder, agoraphobia, social phobia) that are strongly inter-correlated. Recently, a large-scale community sample of adolescents confirmed that the general anxiety disorder (GAD) is a heterogeneous disease that is closely related to other anxiety disorders (Beesdo et al, 2010).

Anxiety and other stress-related disorders are polygenic and complex, rendering the search for mechanisms responsible for the onset of anxiety disorders a challenge. As with most psychiatric diseases, a combination of environmental and genetic factors shape risk and resilience to these disorders (Kendler et al, 1995). Besides meaningful psychodynamic and behavioral concepts, extensive psychoneurobiological research has contributed significantly to the understanding of the disease condition. For example, it was shown that adolescents and adult patients who suffer from anxiety disorders have impaired, hypo-activation of threatsafety discrimination (Britton et al, 2013) which involves maladaptive interactions of the prefrontal cortex in threat perception with the amygdala and hippocampus, parts of the limbic system that form fear responses (fight, flight, or freeze) and emotional memory (Haas and Canli, 2008). While the neural circuits involved in anxiety responses have been dissected in detail, much less is known about the molecular factors contributing to disease.

Genome-wide linkage data and candidate gene as well as genome-wide association studies have indicated some promising genetic loci for anxiety disorders (Erhardt et al, 2011, 2012, ; Howe et al, 2016; Smoller, 2016; Webb et al, 2012), however, confirmed hits across large cohorts, as they now exist for schizophrenia, are missing. As with other psychiatric disorders, large consortium-based studies will be necessary to identify reliable associations. Heritability for anxiety disorders is estimated to range from 30 to $40 \%$, complemented mostly by individually specific environmental factors, such as adverse life events, with the possibility of gene $\times$ environment interactions to strongly contribute to disease risk (Shimada-Sugimoto et al, 2015; Swartz et al, 2017). Epigenetic mechanisms offer a possible molecular integration of both genetic and environmental factors (Klengel and Binder, 2015). Epigenetic regulations refer to mechanisms that alter the accessibility of DNA to transcriptional regulation without affecting the DNA sequence and may include specific sets of histone modifications as well methylation (the addition of a methyl $\left(-\mathrm{CH}_{3}\right)$ group) to cytosine within cytosine-phosphate-guanine $(\mathrm{CpG})$ dinucleotides. Both genetic and environmental factors, including adverse life events, have been shown to have an impact on DNA methylation thus providing a link between these two potential risk domains.

DNA methylation is mainly tissue-specific, nonetheless, sites with cross tissue relevance exist. Consequently, many studies have investigated peripheral tissue, including circulating blood cells, even though the target disease organ is different. For a number of psychiatric disorders, including anxiety disorders, immune-related factors may also contribute directly to pathogenesis. Genome-wide DNA methylation patterns in peripheral blood have been used in the search for novel disease-associated loci in a number of psychiatric disorders, including schizophrenia, post-traumatic stressdisorder (PTSD), and depression (El-Sayed et al, 2012; Nestler et al, 2015). In fact, a recent epigenome-wide association study (EWAS) in peripheral blood in schizophrenia was able to identify differentially methylated loci that overlap with previously identified genome-wide significant single-nucleotide polymorphism (SNP) loci (Montano et al, 2016). So far, however, no large-scale DNA methylation study for anxiety-related traits has been published.

For this reason the current study was undertaken to compare genome-wide $\mathrm{CpG}$ methylation patterns in individuals with generalized anxiety disorder $v s$ healthy participants in a population representative sample of community dwelling adults. The initial epidemiological findings were confirmed in an additional clinical cohort and the functional annotation of the associated sites was extended in a translational animal model for anxiety and stress-relevant stimulation. DNA methylation studies in peripheral blood may thus provide an additional tool to identify molecular risk markers for anxiety disorders.

\section{MATERIALS AND METHODS}

\section{Population-Representative KORA Sample and Cross-Sectional Study Design}

Data were obtained from the KORA (Cooperative Health Research in the Region of Augsburg) F4 study (2006-2008), a follow-up study of the fourth KORA survey (S4) conducted in 1999-2001. The cross-sectional, population-based KORA S4 study sample was recruited from the city of Augsburg and the two adjacent counties in the south of Germany (Holle et al, 2005). The F4 study was restricted to subjects aged 3281 years at follow-up. Of the 4261 participants of the S4 survey, 3080 were examined again in the F4 study. Individuals $(n=1814)$ were randomly selected for genetic and epigenetic analyses (Wichmann et al, 2005; Zeilinger et al, 2013). The assessment of anxiety was based on a selfadministered questionnaire which was sent only to participants aged 32-72 years $(n=1596)$. Among them, 1522 individuals $(95.4 \%$ of those with methylation measurements and aged 32-72 years) had complete data for dependent and independent variables and were included in this analysis.

The study has been conducted according to the principles expressed in the Declaration of Helsinki. Written informed consent has been given by each participant. The study, including the protocols for subject recruitment and assessment and the informed consent for participants, was reviewed and approved by the local ethical committee (Bayerische Landesärztekammer).

\section{Exposure Assessment in KORA}

The Generalized Anxiety Disorder (GAD-7), a 7-item instrument, was used to assess fear and anxiety based 
symptoms that identify the most important diagnostic criteria for Generalized Anxiety according to the DSM-IV, and was assessed by a self-administered questionnaire. This is a valid and reliable tool applied in both clinical and research settings with predefined cutoff values that are wellestablished to assess anxiety (Spitzer et al, 2006). The scale ranges from 0 to 21 , with suggested cutoffs set to distinguish no or minimal anxiety (0-4) from mild (5-9), moderate (10-14), or severe (15-21) anxiety disorder, following Spitzer et al (2006).

\section{Lifestyle and Clinical Confounders in KORA}

Information about daily alcohol intake was gathered in the personal interview and reported in g/day. Smoking status included the categories (regular/occasional/former/never smoker). Standardized measurements of height and weight were obtained during the physical examination and then body mass index $\left(\mathrm{BMI}=\mathrm{kg} / \mathrm{m}^{2}\right)$ was calculated.

\section{Inflammatory Markers in KORA}

Fasting EDTA plasma samples stored at $80^{\circ} \mathrm{C}$ were used to measure concentrations of hsCRP using nephelometry on a $\mathrm{BN}$ II using reagents from Siemens (Eschborn, Germany) and IL-18 by Elisa technology using the IL-18 test kit from MBL (Nagoya, Japan). More details are previously published (Herder et al, 2013). Eighty-six participants were excluded from this analysis due to missing values; however, the number of participants with severe anxiety remained the same. Both, CRP and IL-18 concentrations were approximately lognormal distributed and therefore log-transformed to be used as continuous variables.

\section{Array-Based DNA Methylation Analysis in KORA with Infinium Methylation $450 \mathrm{~K}$}

The blood sampling was conducted by the KORA study staff in Augsburg, Germany. Genomic DNA $(1 \mu \mathrm{g})$ from 1814 samples was bisulfite converted using the EZ-96 DNA Methylation Kit (Zymo Research, Orange, CA, USA) according to the manufacturer's protocol, with the

Table I Population Description in KORA F4 $(n=1522)$

\begin{tabular}{lc}
\hline Characteristics & Distribution \\
\hline Male sex $(n=734, \%)$ & 48.2 \\
Age (years) & $58.6(7.5)$ \\
Smoking $(n=2 \mid 4, \%)$ & $|4|$. \\
Alcohol intake (g/day) & $7.9(22.9)$ \\
BMI (in kg/m²) & $28.0(4.8)$ \\
& \\
GAD-7 Score & 63.1 \\
No/minimal anxiety $(0-4)(n=96 \mid, \%)$ & 29.4 \\
Mild (5-9) $(n=448, \%)$ & 6.0 \\
Moderate $(|0-| 4)(n=9 \mid, \%)$ & 1.5 \\
Severe $(|5-2|)(n=22, \%)$ & \\
\hline aMean (standard deviation). & \\
bMedian (interquartile range). & \\
\end{tabular}

Neuropsychopharmacology alternative incubation conditions recommended when using the Illumina Infinium Methylation Assay. Genome-wide DNA methylation ( $n=485000 \mathrm{CpG}$ sites) was assessed using the Illumina HumanMethylation450 BeadChip as described elsewhere (Pfeiffer et al, 2015). The percentage of methylation of a given cytosine is reported as a $\beta$-value, which is a continuous variable between 0 and 1 , corresponding to the ratio of the methylated signal over the sum of the methylated and unmethylated signals plus an offset of 100 . $M$-values were calculated as the binary logit of the corresponding $\beta$ value. Serum composition of six white blood cell types were estimated using 473 of the 500 most cell-specific CpG sites present on the $450 \mathrm{k}$ array based on the protocol proposed by Houseman et al (2012). The estimated blood cell types include $\mathrm{CD}^{+} \mathrm{CD}^{+}$T-lymphocytes, $\mathrm{CD}^{+} \mathrm{CD}^{+}{ }^{+}$-lymphocytes, $\mathrm{CD}^{+} 6^{+}$Natural Killer cells, CD $19^{+}$B-lymphocytes, $\mathrm{CD} 14^{+}$monocytes, and $\mathrm{CD} 15^{+}$granulocytes.

\section{Data Pre-Processing and Initial Quality Assessment in KORA}

GenomeStudio (version 2011.1) with methylation module (version 1.9.0) was used to extract the raw methylation data generated by BeadArray Reader. Data were preprocessed using R version 3.0.1 (http://www.r-project.org/). All details for preprocessing and references for $\mathrm{R}$ packages used in this study are provided in Supplementary text and Supplementary Table 1. Probes with signals from less than three functional beads and probes with a detection $p$-value $>0.01$ were defined as low-confidence probes. Potential batch or plate effects were accounted for in the regression analyses (see under 'Statistical analyses' below).

\section{MPIP Case-Control Study}

The replication sample was derived from patients from the anxiety disorders outpatient unit and control subjects recruited at the Max Planck Institute of Psychiatry (MPIP) in Munich (Erhardt et al, 2011). Panic disorder was diagnosed using the Structural Clinical Interview for DSM IV (SCID). All patients included in the current analyses were not taking any psychotropic medications. In all patients, severity of panic symptoms was assessed with the Panic and Agoraphobia scale (PAS) (Bandelow, 1995). Control subjects were recruited from a Munich-based community sample after verifying for the absence of axis I psychiatric disorders using the Munich version of the Composite International Diagnostic Interview (M-CIDI) (Wittchen, 1997). All subjects were Caucasian and provided written informed consent. Procedures were approved by the Ethics Committee of the Ludwig Maximilians University, Munich, Germany, in accordance with the Declaration of Helsinki.

\section{DNA Methylation Arrays in the MPIP Case-Control Study}

Genomic DNA was extracted from peripheral blood using the Gentra Puregene Blood Kit (Qiagen). DNA quality and quantity was assessed by a NanoDrop 2000 Spectrophotometer (Thermo Scientific) and Quant-iT Picogreen (Invitrogen). Genomic DNA was bisulfite converted using the Zymo EZ-96 DNA Methylation Kit (Zymo Research) and 
DNA methylation levels were assessed for $>480000 \mathrm{CpG}$ sites using the Illumina HumanMethylation450 BeadChip array. Hybridization and processing was performed according to the instructions of the manufacturer.

Quality control of methylation data including intensity read outs, normalization, cell type composition estimation, $\beta$ - and $M$-value calculation were done using the Bioconductor $\mathrm{R}$ package minfi (version 1.10.2). Failed probes were excluded based on a detection $p$-value larger than 0.01 in $>50 \%$ of the samples ( $n=236$ probes). $\mathrm{X}$ chromosome, $\mathrm{Y}$ chromosome, and nonspecific binding probes were removed (Chen et al, 2013). We also excluded probes if SNPs were documented in the interval for which the Illumina probe is designed to hybridize. We also removed probes if they were located close (10 bp from query site) to an SNP which had a minor allele frequency of $\geqslant 0.05$. The data were then normalized with functional normalization (Fortin et al, 2014), an extension of quantile normalization included in the $\mathrm{R}$ package minfi. Batch effects were identified by inspecting the association of the first principal component of the methylation levels with bisulfite conversion plate, well, slide, slide row, and array using linear regression and visual inspection of PCA plots using the Bioconductor R package shinyMethyl (version 0.99.3). In order to remove batch effects, the Empirical Bayes' (EB) method ComBat was used.

\section{Murine Model of Acute Social Defeat Stress and Gene Expression in Mouse Blood}

To examine whether the Asb1 gene is responsive to acute stress exposure, we used an acute model of social defeat stress in mice, which is relevant for anxiety-related phenotypes (Campos et al, 2013). Adult C57BL/6N male mice (3-4 months old) were exposed to acute social defeat stress as previously described (Wang et al, 2013). Briefly, mice were divided into a control $(n=7)$ and a stress $(n=9)$ group. Stressed mice were introduced into the home cage of an aggressive CD1 resident mouse for $5 \mathrm{~min}$. Mice were allowed to interact without any interference from the experimenter. In the case that an animal was injured, the experimenter intervened immediately and the mouse was excluded from analysis. Control mice were allowed to explore an empty novel cage, identical to the resident cage, for $5 \mathrm{~min}$. Exactly $4 \mathrm{~h}$ following stress/control exposure, mice were anesthetized with isoflurane and killed. Trunk blood was harvested to assess levels of corticosterone (in order to validate the stress paradigm) as well as to assess gene expression using the PAXgene method.

For assessment of gene expression, $250 \mu \mathrm{l}$ of trunk blood was placed in PAXgene Blood RNA Tubes (PreAnalytiX). RNA sample quality was qualitatively analyzed using capillary gel electrophoresis chip (RNA 6000 Nano Kit; Agilent, Boeblingen, Germany). RNA samples of sufficient quality $(\mathrm{RIN} \geqslant 7.5)$ were then processed with the Illumina TotalPrep-96 RNA Amplification Kit (Ambion, Austin, TX, USA) and hybridized onto MouseWG-6 v2 Expression BeadChips (Illumina, San Diego, CA, USA).

For corticosterone measurements, trunk blood was collected in EDTA-coated microcentrifuge tubes (Kabe Labortechnik, Nuembrecht-Elsenroth, Germany). Plasma was isolated following centrifugation at $6000 \mathrm{~g}$ at $4{ }^{\circ} \mathrm{C}$ for $15 \mathrm{~min}$. A commercially available radioimmunoassay kit with ${ }^{125}$ I-labeled anti-corticosterone antibody was used to determine plasma corticosterone levels (MP Biomedicals, Solon, OH, USA; sensitivity $12.5 \mathrm{ng} / \mathrm{ml}$ ).

\section{Statistical Analyses}

KORA study. To assess unadjusted associations between generalized anxiety categories and characteristics, chi-squared tests, and F-test or Kruskal-Wallis tests were performed for associations with categorical and continuous characteristics, respectively. To assess adjusted associations between generalized anxiety and methylation, that is, taking potential confounders into account, linear mixed effects models were used with methylation $M$-values as response variable, anxiety as exposure variable and sex, age, and white blood cell proportions based on constrained projection (Houseman et al, 2012) as additional covariates to adjust for potential confounding. In KORA, sensitivity analyses were conducted with BMI, smoking, and alcohol consumption included as additional covariates. KORA F4 samples were processed on 20 96-well plates in nine batches; plate and batch effects were investigated using principle component analysis and eigenR2 analysis. The plate variable explained $4.8 \%$ (F4) of variance in the DNA methylation data. Consequently, plate was included as a random effect in the analyses. This approach was applied to individuals categorized by the GAD-7 anxiety instrument and mild, moderate, or severe anxiety were each compared to no or minimal anxiety. We did not use the GAD-7 score as a continuous variable as this approach would require an assumed linear relation between anxiety and methylation; nonetheless, our analyses showed a similar methylation pattern for the lowest three anxiety categories and a comparatively higher $M$-value for the severe anxiety category. Effect estimates are given as $\beta$ coefficient with standard error (SE) drawn from the linear mixed effects models comparing mean $M$-values of mild, moderate, or severe anxiety to the reference category no or minimal anxiety.

Analyses that considered the influence of inflammatory markers on the observed association between level of anxiety and methylation were run using dummy variables for each anxiety level in order to model the full cohort. Four CpG sites were assessed (cg12701571, cg21650243, cg00708580, and cg14380745) to test for an interaction between anxiety level and inflammation on methylation of the Asb1 gene. Finally, adjusted mean levels of inflammatory markers were assessed in sex-stratified analyses of participants with severe $v s$ no or minimal anxiety. Generalized linear models included age, BMI, smoking status, and alcohol consumption.

We used $M$-values as the outcome in the linear mixed effects models, since it shows better statistical ability compared to $\beta$-values and a normal distribution can be assumed (Du et al, 2010). The nlme package in $\mathrm{R}$ was used to run the linear mixed effects models over the $450 \mathrm{~K}$ array and proc glm and proc mixed in SAS9.4 (Cary, NC) were used to run the inflammatory marker analyses.

For those CpG sites which were significantly associated with methylation $M$-values, potential differences in the association between men and women was assessed with an 'interaction model' by adding respective interaction terms to the model described above and estimating the respective $p$-values for interaction. An analysis stratified by sex was 
then performed to obtain the effect estimates for males and females separately.

The statistical package $\mathrm{R}$ version 3.0.1 was used to run the EWAS, and SAS 9.2 (NC, USA) for Windows was used for the specific sub-analyses. $p$-Values were assessed as uncorrected and corrected for multiple testing by the Bonferroni approach (significance level 0.05/441,552-1.1E-07).

MPIP anxiety study. A linear regression model was fitted for cg12701571 with the batch corrected $M$-values after ComBat to test for a case $v s$ control difference and for the association of methylation levels with anxiety symptoms within cases. Sex, age, and cell composition (Houseman et al, 2012) were included as covariates in all regression models.

Murine model of acute social defeat stress. Data were analyzed using R 3.2 (used package: beadarray) and Omics Explorer 3.1 (Qlucore). Values are presented as technical batch-corrected residuals.

\section{RESULTS}

\section{Demographic, Biomedical and Psychological Characteristics of the KORA F4 Population}

Table 1 gives an overview about the demographic, lifestyle, and clinical characteristics of the study population $(n=1522)$. Men and women were almost equally distributed (48.2 and 51.2\%); mean age was 58.6 years (standard deviation 7.5 , range $32-72$ years).

Approximately one-quarter of the study participants were classified with mild anxiety (26.2/32.5\% for men/women), $3.5 / 8.3 \%$ with moderate, and $1.6 / 1.3 \%$ with severe anxiety. Anxiety was more pronounced in women than in men $(p<0.001)$. Age and anxiety were not significantly associated in the KORA cohort ( $p$-value 0.867). Moreover, no significant associations were found for BMI, smoking, alcohol use, or white blood cell count with anxiety (Supplementary Table 2). The overall $p$-value for anxiety $\times$ smoking was 0.47 , trend test 0.18 . Even testing only severe against no/minimal was not NS $(p=0.22)$. The distributions of white blood cell composition were rather similar in the four anxiety categories; $p$-values were between 0.020 (natural killer cell) and 0.476 (B cell) subsets indicating no significant association after Bonferroni correction (adjusted $p=0.05 / 6$ $=0.008)$.

\section{Increased CpG Methylation in the Promoter Region of} Asb1 Associates with Severe Anxiety in the KORA Study

We analyzed DNA methylation to identify CpG sites associated with severe, moderate, or mild anxiety compared to healthy participants, that is, participants with no or minimal anxiety. No significant association was found for mild or moderate anxiety. However, in comparisons between severe and no or minimal anxiety, we identified a single CpG site $(\mathrm{cg} 12701571)$ in the promoter region of the ankyrin repeat and SOCS box protein 1 (Asb1) gene located on chromosome 2q37, with differential methylation levels after conservative correction for multiple testing $(p \leqslant 1 E-07)$ throughout the genome.

Table 2 presents the detailed effect estimates calculated for each of the anxiety categories with uncorrected and corrected $p$-values. The increase in methylation $M$-values at the $\mathrm{CpG}$ cg12701571 for severe compared to no or minimal anxiety was 0.56 (SE 0.10, $p$-value Bonferroni $=0.005$ ). This difference remains significant even if corrected further for the number of comparisons ( $p$-value after Bonferroni correction of $0.015 \quad(=0.005 \times 3)$. A sensitivity analysis adjusted additionally for lifestyle factors (BMI, smoking, and alcohol) revealed very similar estimations for cg12701571 ( $\beta$-coefficient $=0.56, \mathrm{SE}=0.10, p$-value Bonferroni $=0.005)$. In two other sites in the same region (cg08341874 and cg21593923), borderline significant associations with anxiety was also observed ( $p$-values 0.041 and 0.048). These associations of anxiety with cg08341874 and cg21593923 lost significance after lifestyle factors were taken into account. Investigation of genetic confounding was carried out to identify whether the observed associations between anxiety and methylation levels were due to SNPs in the $A s b 1$ region (Figure 1). We tested all SNPs in the Asb1 region for association with DNA methylation levels and anxiety in the KORA F4 data set. None of the tested SNPs were nominally associated $(p<0.05)$ with either anxiety or methylation.

To display the actual methylation values and differences, the unadjusted median $\beta$-value methylation for each anxiety category and their \%-change compared to no or minimal anxiety are additionally provided and showed a \%-change in median $\beta$-value methylation of $48.5 \%$. A stratified analysis

Table 2 Association of Anxiety and cg|270 I57| Asb I $(n=1522)$ Estimated by Linear Mixed Effects Model and Unadjusted Median $\beta$-Value Methylation with \%-Change Compared to No or Minimal Anxiety in KORA F4

Anxiety category $\beta$-coefficient SE uncorrected $p$-Value uncorrected $p$-Value Bonferroni Median $\beta$-value Median \%-change in $\beta$-value

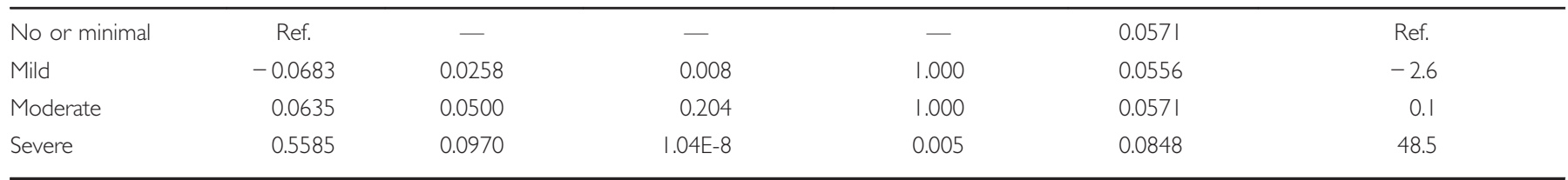

The $\beta$-coefficients with respective standard error (SE) estimated by a linear mixed effects model for the association of anxiety (explanatory variable) and $M$-value

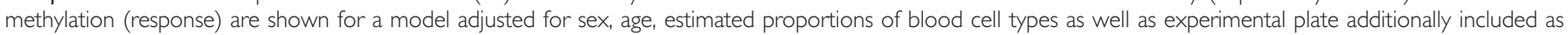
random effect.

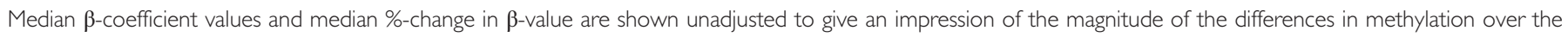
anxiety categories. 


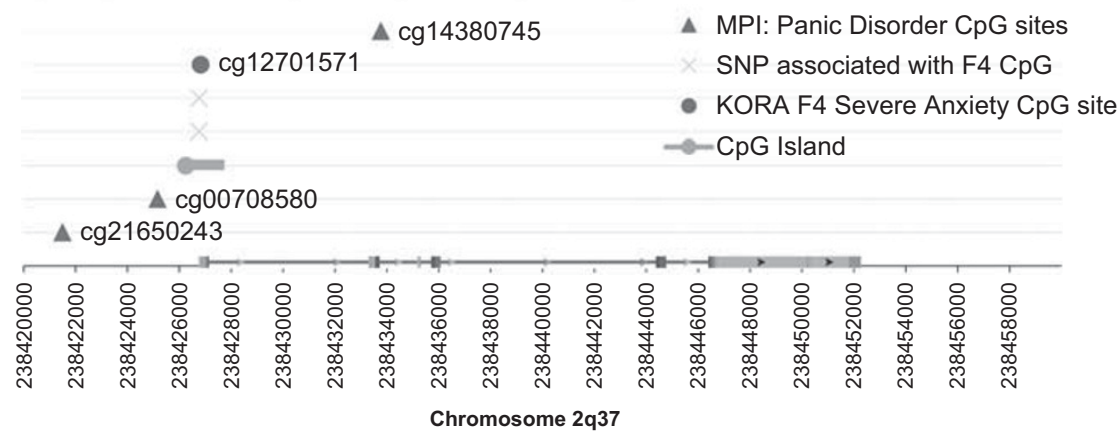

Figure I Genetic map of the Asb / gene locus on human chromosome 2 indicating the CpG and SNP locations under investigation. From http://www.ncbi. nlm.nih.gov/gene/5I 665.

revealed that the effect of increased methylation on this $\mathrm{CpG}$ site was more pronounced for women (0.683 (SE 0.148), $p$-value $<0.0001$ ) as compared to men (0.419 (SE 0.130), $p$-value 0.0013$)$ who suffered from severe anxiety; however, this sex-specific interaction was not significant ( $p$-value for interaction 0.176).

We performed a multinomial logistic regression using the $M$-values of cg12701571 as exposure, anxiety as outcome (with no or minimal as reference), and adjustment for age, sex, and white blood cell proportions. This analysis revealed similar findings with a significant association for having severe compared to no or minimal anxiety (Supplementary Table 3); the ORs for one unit increase of the $M$-value was 6.56 (95\% CI 3.00-14.32, $p$-value <0.0001).

\section{An Interaction Between IL-18 and Anxiety is Associated with Asb1 Methylation}

A tendency towards a significant interaction between IL-18 and severe anxiety on methylation changes at $\mathrm{CpG}$ site cg12701571 was observed in the total population $(p=0.08)$ and amoung women, a significant interaction was observed $(p=0.014)$ (see Table 3a). Interestingly, one of the other CpG sites (cg21650243) upstream of this CpG of interest showed significant interactions with IL-18 levels in mildly anxious participants (total, $p=0.052$; women $p=0.007$ ) but here, mild anxiety was inversely associated with methylation, in the total population and in women (Table 3b). Adjusted mean values of IL-18 were significantly reduced in severely anxious women compared to those with minimal to no anxiety (Table 4).

Models that accounted for CRP levels showed opposing estimated effects for mild and severe anxiety on the methylation of $\mathrm{cg}$ cg12701571. In the total population as well as for both women and men, severe anxiety showed a positive association with the methylation of this CpG site whereas mild anxiety was negatively associated with methylation of this site, predominantly in women (Table 3a). Changes in cg21650243 methylation were not influenced by anxiety level with adjustment for CRP (Table 3b).

\section{Asb1 Methylation is Associated with Panic Symptom Severity in the MPIP Study}

Using Illumina $450 \mathrm{~K}$ DNA methylation array data for cg12701571, we then examined whether these findings replicated in the independent MPIP study. We observed no differences in cg12701571 methylation levels between patients and controls $(\beta=0.037, \mathrm{SE}=0.025, p=0.146$, Total $n=300)$. However, within cases, and after controlling for age, sex, and blood cell composition, we observed a significant positive association of cg12701571 methylation levels with PAS scores $(\beta=0.005, \mathrm{SE}=0.002, p=0.021, n=131)$ (Figure 2). Three other $\mathrm{CpG}$ sites showed significant differences between panic cases and controls (cg21650243: $\beta=0.323, \quad \mathrm{SE}=0.162$, $p=0.047 ; \quad \operatorname{cg} 00708580: \quad \beta=0.490, \quad \mathrm{SE}=0.191, \quad p=0.011$; cg14380745: $\beta=0.477, \mathrm{SE}=0.238, p=0.046)$.

\section{Effects of Acute Social Defeat Stress on Asb1 Expression Levels in a Murine Model}

Like other stress-related phenotypes, anxiety disorders have been associated with DNA methylation changes in stressresponsive genes (Klengel et al, 2013; Zannas et al, 2015). Thus, it is plausible that the $A s b 1$ methylation changes, which we observed in subjects with severe anxiety, could be attributed to the responsivity of the $A s b 1$ gene to stress exposure. To address this hypothesis, we compared Asb1 mRNA levels in the peripheral blood of adult mice exposed to acute social defeat stress $(N=9)$ with control (non-stressed) mice $(N=7)$. The effectiveness of this stress paradigm to elicit a stress response was confirmed by the increased plasma corticosterone (CORT) levels in response to stress $\left(\mathrm{CORT}_{\text {control }}=24.81\right.$ vs $\mathrm{CORT}_{\text {stress }}=71.39$ : $p=0.017)$. Among the four probes covering Asb1 in our gene expression array data, two probes showed significant stress-induced upregulation (ILMN_2430887: $p=0.006$; ILMN_1233302: $p=0.016$ ), and ILMN_2430887 remained significant after Bonferroni correction for multiple comparisons (adjusted $p=0.05 / 4=0.013$; Figure 3).

To understand whether the response of $A s b 1$ to stress is tissue-specific, we further examined if stress had a similar effect on the $A s b 1$ expression levels measured with the ILMN_2430887 probe in the brain amygdala, hippocampus, prefrontal cortex, and hypothalamus. Asb1 expression was not responsive to stress in any of these brain regions $(p>0.05)$. These findings demonstrate Asb1 shows tissue-specific response to stress in the mouse peripheral blood. Because ASB1 belongs to the SOCS family of proteins that are thought to play important roles in inflammation (Elliott and Johnston, 2004), we then examined whether Asb1 levels correlate with the expression of the 
Table 3a Linear Mixed Effects Models of Cytokine and Anxiety Associations with cg I270 I57I CpG Methylation in KORA F4

\begin{tabular}{|c|c|c|c|c|c|c|c|c|c|}
\hline & \multicolumn{3}{|c|}{ Total } & \multicolumn{3}{|c|}{ Men } & \multicolumn{3}{|c|}{ Women } \\
\hline & Estimate & SE & $p$-Value & Estimate & SE & $p$-Value & Estimate & SE & $p$-Value \\
\hline \multicolumn{10}{|l|}{ CRP } \\
\hline \multicolumn{10}{|l|}{ Model I } \\
\hline Mild anxiety & -0.139 & 0.058 & 0.017 & -0.018 & 0.083 & 0.826 & -0.212 & 0.081 & 0.009 \\
\hline Severe anxiety & 0.691 & 0.216 & 0.001 & 0.657 & 0.284 & 0.021 & 0.695 & 0.322 & 0.031 \\
\hline Log CRP & 0.015 & 0.025 & 0.535 & 0.030 & 0.035 & 0.396 & 0.008 & 0.035 & 0.831 \\
\hline \multicolumn{10}{|l|}{ Model 2} \\
\hline Mild anxiety & -0.145 & 0.060 & 0.015 & -0.019 & 0.084 & 0.820 & -0.224 & 0.084 & 0.008 \\
\hline Moderate anxiety & 0.017 & 0.116 & 0.882 & -0.016 & 0.197 & 0.936 & -0.011 & 0.145 & 0.938 \\
\hline Moderate $\times$ Log CRP & -0.023 & 0.096 & 0.812 & 0.104 & 0.154 & 0.497 & -0.098 & 0.124 & 0.431 \\
\hline Severe $\times$ Log CRP & -0.360 & 0.235 & 0.125 & -0.317 & 0.266 & 0.234 & -0.548 & 0.459 & 0.233 \\
\hline \multicolumn{10}{|l|}{ IL-18 } \\
\hline \multicolumn{10}{|l|}{ Model I } \\
\hline Mild anxiety & -0.143 & 0.058 & 0.015 & -0.022 & 0.083 & 0.788 & -0.213 & $0.08 I$ & 0.009 \\
\hline Moderate anxiety & 0.012 & 0.113 & 0.913 & 0.008 & 0.195 & 0.969 & -0.047 & 0.140 & 0.737 \\
\hline Severe anxiety & 0.683 & 0.216 & 0.002 & 0.672 & 0.285 & 0.019 & 0.691 & 0.324 & 0.033 \\
\hline Log IL-18 & -0.070 & 0.065 & 0.280 & -0.134 & 0.098 & 0.171 & -0.019 & 0.087 & 0.829 \\
\hline \multicolumn{10}{|l|}{ Model 2} \\
\hline Mild anxiety & -0.888 & 0.780 & 0.256 & -0.452 & 1.249 & 0.717 & -0.942 & 1.050 & 0.370 \\
\hline
\end{tabular}

Linear mixed effects model for the association of inflammatory markers (CRP top, IL-I 8, bottom), anxiety (explanatory variable using dummy variables for mild moderate and sever anxiety) and M-value cgl270 I57/ methylation (response) are shown for a model adjusted for sex, age, estimated proportions of blood cell types as well as experimental plate additionally included as random effect (Model I). Model 2 included interaction terms for anxiety level and cytokine. Statistical significance at $p<0.05$ is indicated in bold.

proinflammatory cytokines interleukin-6, tumor necrosis factor, and interleukin 1 beta (IL-1 $\beta$ ). Among these, only the $I L 1 b$ gene was expressed above background in our mouse blood arrays. IL $1 b$ expression robustly correlated with Asb1 mRNA levels $(r=0.567, p=0.022)$ and was significantly induced by stress exposure $(p<0.001)$. Together, these findings show that $A s b 1$ is associated with a stressinduced proinflammatory gene expression profile in peripheral blood.

\section{DISCUSSION}

This is the first large-scale, population-based EWAS in anxiety published to date, and demonstrates increased methylation in the promoter region of the $A s b 1$ gene with severe symptoms of anxiety in participants from the KORA
Study in Augsburg (Southern Germany). Moreover, this finding of anxiety-related hyper-methylation was supported in a clinical sample of patients with anxiety symptoms derived from the Anxiety Disorders Outpatient Clinic of the Max Planck Institute of Psychiatry (MPIP) (Erhardt et al, 2011). The Asb1 transcript was shown to be responsive to acute social defeat stress in mice specifically in peripheral blood, suggesting that ASB1 regulation may be related to environmental risk factors for anxiety.

In both cohorts, more severe anxiety symptoms were associated with an increased methylation of a single $\mathrm{CpG}$, cg12701571. This CpG is located upstream of the transcription start site of $A s b 1$ within a site showing histone marks (H3K27 acetylation) indicative of an active enhancer. At the same location, chromatin-histone immune precipitation studies indicate binding of the zinc-finger transcription factors Early Growth Response (EGR) 1, EGR2, EGR3, and 
Table 3b Linear Mixed Effects Models of Cytokine and Anxiety Associations with cg21650243 CpG Methylation in KORA F4

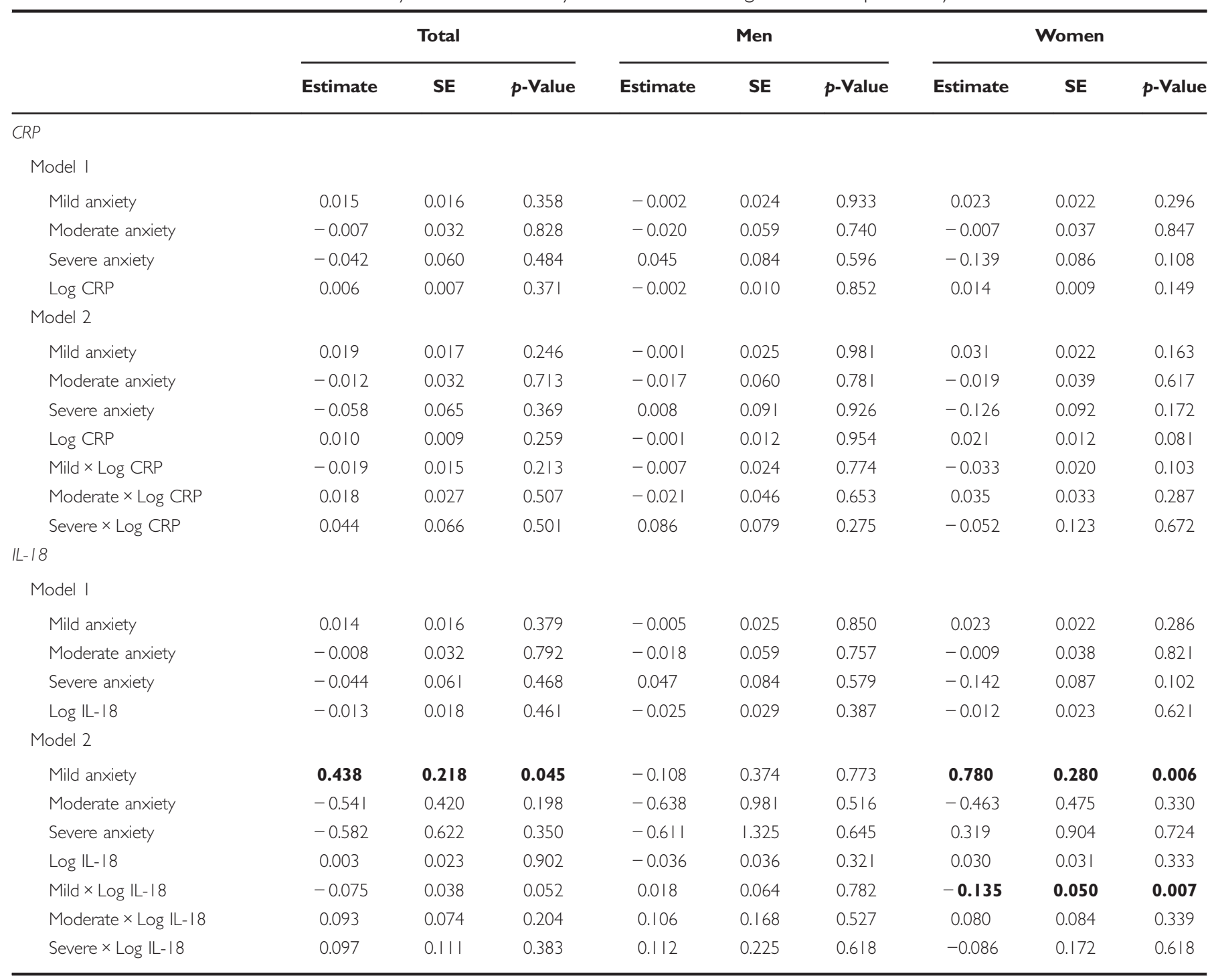

Linear mixed effects model for the association of inflammatory markers (CRP top, IL- I 8, bottom), anxiety (explanatory variable using dummy variables for mild moderate and severe anxiety) and M-value of cg21650243 methylation (response) are shown for a model adjusted for sex, age, estimated proportions of blood cell types as well as experimental plate additionally included as random effect (Model I). Model 2 included interaction terms for anxiety level and cytokine. Statistical significance at $p<0.05$ is indicated in bold.

EGR4. The location of this $\mathrm{CpG}$ is suggestive of a possible functional role in transcriptional regulation of $A s b 1$.

The Asb1 gene is located on chromosome 2q37, and encodes a protein with 335 amino acids. The ASB1 protein is a member of the ankyrin repeat and SOCS box-containing (ASB) family of proteins and is expressed in a number of tissues, including blood cells and brain. The methylation at this specific $\mathrm{CpG}$ shows some trend for a positive correlation between blood and the tissue from the prefrontal cortex with $r=0.209$ and $p=0.07$ (http://epigenetics.essex.ac.uk/bloodbrain/?probenameg $=\operatorname{cg} 12701571)$.

While the ASB protein family (1-18) is the largest class of SOCS box proteins, they remain relatively uncharacterized (Muniz et al, 2013). ASB1 associates with several cullinassociated neddylation proteins that are involved in the targeted ubiquitin degradation pathways (Andresen et al, 2014). The ASB1 protein contains ankyrin repeats that provide scaffolding support and facilitate structure in neuronal tissue and have been shown to be involved in neurogenesis and neuroprotection (Leussis et al, 2012). In muscle, ankyrin repeat proteins are involved in stress and injury-induced repair and regeneration (Miller et al, 2003). Another ankyrin repeat gene ANK3 has been associated in a GWAS with bipolar disorder (Leussis et al, 2012) and a genetic polymorphism in ANK3 was recently identified in PTSD patients (Logue et al, 2013). Furthermore, ankyrins as cytoskeletal adaptor proteins interact with sigma-1 receptors, which are unique endoplasmic reticulum proteins that have been implicated in anxiety and depressive-like memory alterations in knockout mouse models (Chevallier et al, 2011).

The other major functional component of ASB1 is the SOCS box which is known to partner with the elongin $B$ and $\mathrm{C}$ complexes and thereby also regulate protein turnover by targeting proteins for ubiquitination (Alexander et al, 1999). SOCS proteins have cytokine suppressor functions (Krebs 
Table 4 Least-Squares Mean of CRP and IL-I 8 in Severe vs no Anxiety Categories Stratified by Sex in KORA F4

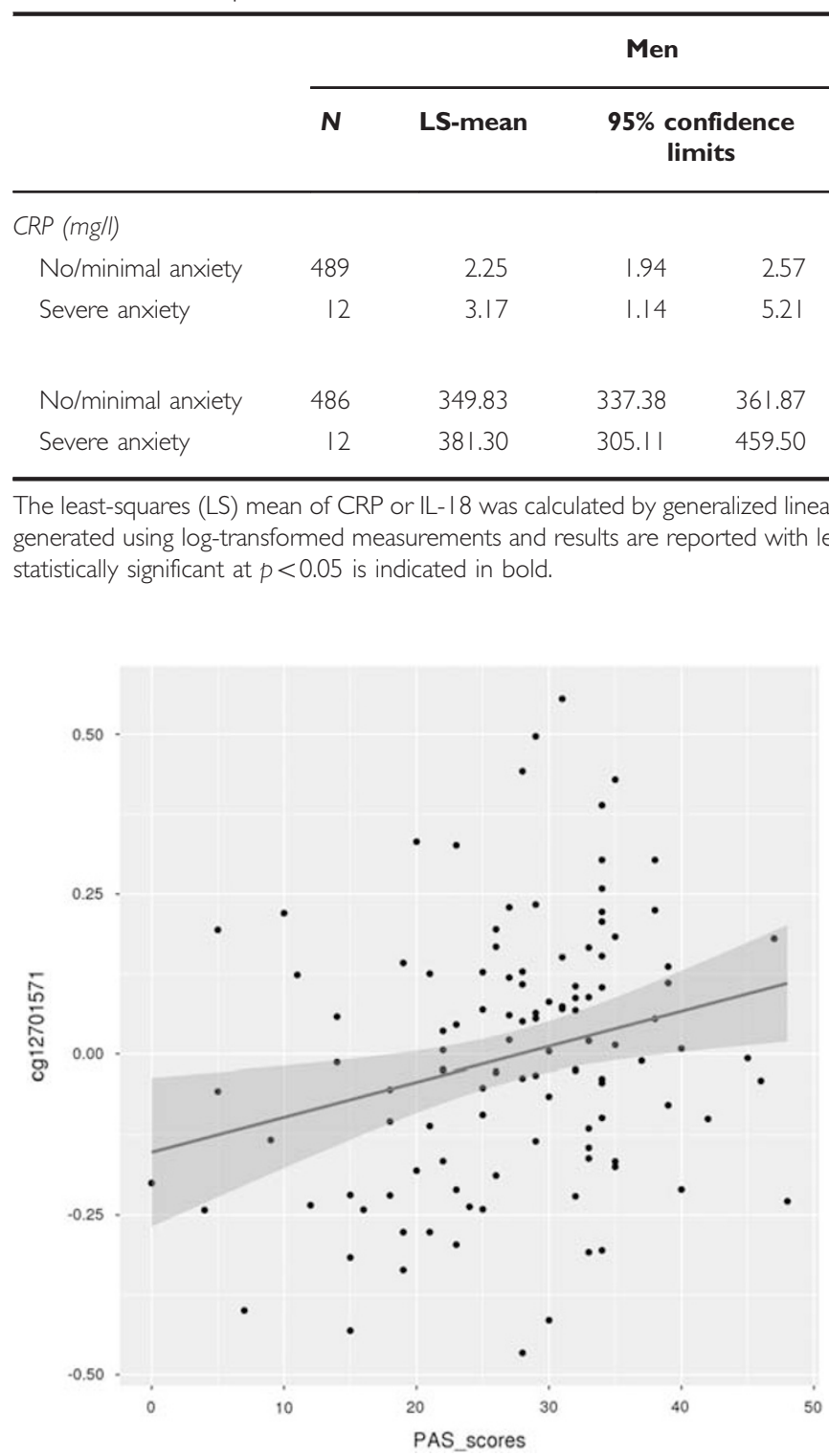

Figure 2 Scatter plot of the correlation between PAS scores in the MPIP cohort $(n=|3|)$ and methylation levels of $c g|270| 57 \mid$. The regression line and $95 \%$ confidence intervals are depicted with the continuous black line and gray shade, respectively.

and Hilton, 2001) and important roles in inflammation, allergy, and homeostasis (Elliott and Johnston, 2004). A link between SOCS proteins and moderation of inflammatory responses is well-established (Boosani and Agrawal, 2015; Marzi et al, 2016), yet the role of inflammatory markers in fear-based disorders is not entirely clear with positive, negative, and null findings prevalent in the literature (Michopoulos et al, 2017). Among women with severe anxiety, we observed reduced serum levels of IL-18 which corroborates a hypothesis of reduced IL-18 due to disregulated reactive oxygen species-related metabolism in patients suffering from trauma (Segman et al, 2005). Polymorphisms in this member of the IL-1 superfamily were shown to be predictive of depression and anxiety through altered amygdala reactivity of threat perception in women but not in men (Swartz et al, 2017). While the role of ASB family members is not well described (Chung et al, 2005), one family member ASB3 has been shown to regulate responses to the pro-inflammatory cytokine tumor necrosis factor (TNF)-alpha by mediating the degradation of its receptor TNF-R2 (Chung et al, 2005) and its gene expression is downregulated in colorectal cancer (Du et al, 2017). The impact of stress-induced Asb1 expression on peripheral inflammation was further supported in our mouse model, where we observed strong correlation of Asb1 expression with expression of the $I L-1 B$ gene. The inflammasome, a multiprotein component of innate immune responses, is suggested to be a key mediator of physiologic and psychological stressors by way of its activation of IL- $1 \beta$ and IL-18, which are involved in neuroinflammation and neurodegeneration (Wong et al, 2016). Thus, altered regulation of $A s b 1$ may impact inflammatory pathways, but whether these peripheral effects are a cause, effect, or epiphenomenon of anxiety phenotypes remains to be examined by future studies.

One of the main risk factors for anxiety disorders are adverse life events. We wanted to assess whether Asb1 is regulated by such environmental risk factors and therefore measured its expression using a mouse model of social defeat stress. These translational studies demonstrated that acute stress response resulted in significant upregulation of the $A s b 1$ gene transcript in peripheral blood of socially defeated mice compared to controls, $4 \mathrm{~h}$ after stress exposure. Together our results may suggest that $A s b 1$ transcription is altered in the acute stress response, as evidenced by the murine model, and that differences in the DNA methylation of that locus are associated with maladaptive stress response conditions in human cohorts. Whether the changes are causally related or the consequence of anxiety disorders cannot be resolved in this study. While this is the first study to identify a role for $A s b 1$ in anxiety disorders, evidence for a role of this relatively unknown gene in other areas of mental health have been demonstrated, for example, in a de novo mutation in individuals with autism spectrum disorder (Neale et al, 2012).

Our results contribute to a few other studies that have identified epigenetic modifications associated with common mood disorders in clinical and smaller population-based settings (El-Sayed et al, 2012). One study of panic disorder 


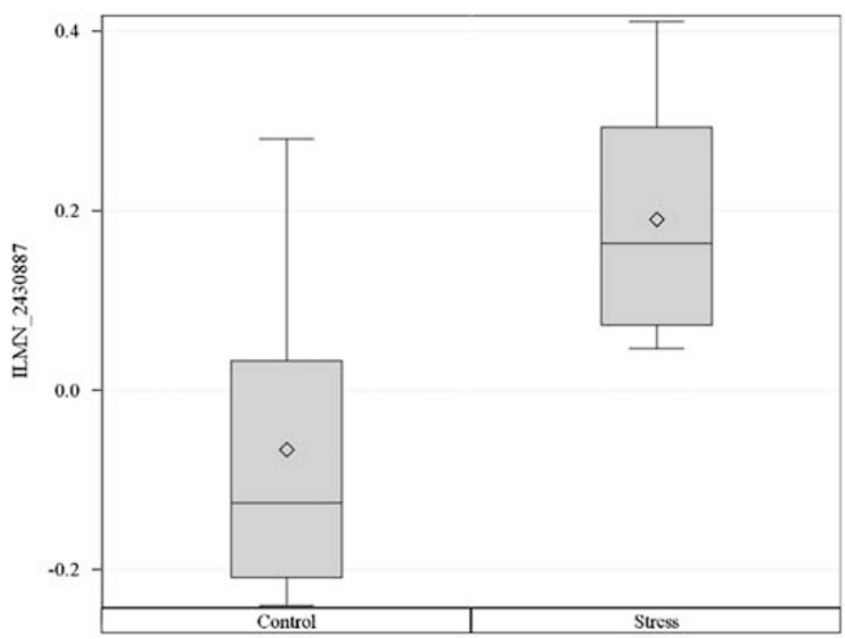

Figure 3 Box plots of Asb / gene expression levels (represented by the ILMN_2430887 probe) detected in mouse blood after social defeat exposure $(n=9)$ or control conditions $(n=7)$. Values in the $y$ axis are presented as batch-corrected residuals.

patients $(n=36)$ has identified reduced function of the noradrenaline transporter (NET) due to increased methylation of the promoter region of this gene (Bayles et al, 2013). A number of genes involved in the innate and adaptive immune system were shown to be differentially methylated between 23 individuals suffering from post-traumatic stress disorder from the Detroit Neighborhood Health Survey and 77 without PTSD (Uddin et al, 2010). A comparison of 50 participants with lifetime PTSD and 60 control participants demonstrated decreased methylation of a neurotransmitter in the opioid system which is also associated with inflammatory pain (Smith et al, 2011). Finally, a small epigenetic study $(n=25$ anxious and $n=22$ non-anxious individuals) showed a relationship between anxiety, epigenetics, and interleukin 6 (IL-6) (Murphy et al, 2015). Taken together, these data add to the mounting evidence of immunologically distinct mechanisms that may underlie pathogenesis of distress-related disorders and contribute population-based insight into epigenetic mechanisms that may contribute to the social threat hypothesis of anxiety, inflammation, and depression (Camacho, 2013; Slavich and Irwin, 2014).

\section{Limitations and Strengths}

The main analysis used data from a population-based study. Individuals are influenced by many different environmental factors and are not subject to specific control criteria. Thus, it is remarkable to find DNA methylation profiles associating with severe anxiety. We showed that applying an EWAS approach to population studies can provide evidence for epigenetic mechanisms that might be connected to distressrelated diseases. The interpretation of results from bloodbased EWAS, in the case of genes involved with immune responses is challenged by the concern that observations may be driven by the cellular composition of the blood samples obtained. The inclusion of cell count estimates in our adjusted analyses is a standard approach used to minimize this bias. Because of the cross-sectional design of this study, we are unable to draw conclusions about the causal nature of the associations observed.

The present study utilized two different questionnaires (GAD-7 and PAS) to measure anxiety symptoms in the included cohorts. To our knowledge, there are no studies examining the correlation between the two questionnaires, and GAD-7 was not available in the MPIP study which is a study focusing on panic symptomatology. The GAD-7 instrument fulfills the criteria of the DSM-5 classification criteria of the American Psychiatric Association, is a good indicator of anxiety symptom severity (Beard and Björgvinsson, 2014), and has a strong overlap with panic disorder (Kroenke et al, 2007). While the approach of using different questionnaires has inherent limitations, GAD-7 and PAS are comparable to the extent that both questionnaires interrogate the common underlying construct of anxiety. Furthermore, hypermethylation in the $A s b 1$ gene was also observed with increasing symptom severity in patients with anxiety disorders which suggests a dose response of stress induced epigenetic changes. Our sampling population was composed of Caucasians of European ancestry, so the generalizability of these results to other ethnicities requires further investigation. The small number of cases $(n=22$ individuals with severe anxiety in the discovery study (KORA F4) vs $n=961$ controls) may have limited the number of significant hits we could identify; nonetheless, our epidemiologic discovery was supported by comparable association in a larger clinical sample of patients, and other published studies of methylation and stress disorders noted above have reported similarly small case samples as our discovery cohort.

Finally, we acknowledge that the differences in study design and cohorts preclude our ability to establish a replication of our epidemiologic finding; however, the supporting clinical study demonstrated that the $A s b 1$ gene is epigenetically modified by more than one fear-associated disorder. Our aim in translating these results into a mouse model was to gain mechanistic insight by examining whether the $A s b 1$ gene is stress regulated, with tissue specificity and functional consequences. Overall, we find the evidence provided warrants future study to further characterize the contribution of epigenetic changes in this relatively unknown gene region that may contribute to inflammatory alterations in fear-based disorders.

\section{CONCLUSIONS}

The present study suggests that the $A s b 1$ gene is responsive to stress exposure and epigenetically modified by anxiety phenotypes. While future studies need to mechanistically dissect the functional relationship between Asb1 methylation and expression and inflammatory consequences in the context of both acute and chronic anxiety, our data suggest that the altered regulation of $A s b 1$ in response to acute stress is specific for peripheral blood and may be relevant for changes in levels of peripheral inflammation. This adds to the mounting evidence of immunologically distinct mechanisms that may underlie pathogenesis of stress-related disorders. Further studies are warranted to characterize the pathogenetic role of $A s b 1$ in anxiety disorders. 


\section{FUNDING AND DISCLOSURE}

None of the authors have biomedical financial interests or potential conflicts of interest to declare. The KORA study was initiated and financed by the Helmholtz Zentrum München-German Research Center for Environmental Health, which is funded by the German Federal Ministry of Education and Research (BMBF) and by the State of Bavaria. Furthermore, KORA research was supported by the portfolio area "metabolic dysfunction" by the Helmholtz Association and within the Munich Center of Health Sciences (MC-Health), Ludwig-Maximilians-Universität, as part of LMUinnovativ. The German Diabetes Center was supported by the Ministry of Science and Research of the State of North Rhine-Westphalia (MIWF NRW) and the German Federal Ministry of Health (BMG). This study was supported in part by a grant from the German Federal Ministry of Education and Research (BMBF) to the German Center for Diabetes Research (DZD). The investigations of the MPIP cohort were supported by ERA-NET NEURON (AnxBio). ASZ is currently supported by a MarieSklodowska Curie fellowship (H2020 grant\# 653240). RTE is supported by The Dartmouth Clinical and Translational Science Institute, under award number UL1TR001086 from the National Center for Advancing Translational Sciences (NCATS) of the National Institutes of Health (NIH).

\section{ACKNOWLEDGMENTS}

We are grateful for the commitment and involvement of all the study participants and for the work and dedication of the MONICA/KORA Augsburg Study staff. We thank Nadine Lindemann and Franziska Scharl for technical support in the DNA methylation measurements that were performed at the Helmholtz Zentrum München.

\section{REFERENCES}

Alexander WS, Starr R, Metcalf D, Nicholson SE, Farley A, Elefanty AG et al (1999). Suppressors of cytokine signaling (SOCS): negative regulators of signal transduction. J Leuk Biol 66: 588-592.

Andresen CA, Smedegaard S, Sylvestersen KB, Svensson C, Iglesias-Gato D, Cazzamali G et al (2014). Protein interaction screening for the ankyrin repeats and suppressor of cytokine signaling (SOCS) box (ASB) family identify Asb11 as a novel endoplasmic reticulum resident ubiquitin ligase. J Biol Chem 289: 2043-2054.

Bandelow B (1995). Assessing the efficacy of treatments for panic disorder and agoraphobia. II. The Panic and Agoraphobia Scale. Int Clin Psychopharmacol 10: 73-81.

Bayles R, Baker EK, Jowett JB, Barton D, Esler M, El-Osta A et al (2013). Methylation of the SLC6a2 gene promoter in major depression and panic disorder. PLoS ONE 8: e83223.

Beard C, Björgvinsson T (2014). Beyond generalized anxiety disorder: psychometric properties of the GAD-7 in a heterogeneous psychiatric sample. J Anxiety Disord 28: 547-552.

Beesdo K, Pine DS, Lieb R, Wittchen H (2010). INcidence and risk patterns of anxiety and depressive disorders and categorization of generalized anxiety disorder. Archiv Gen Psychiatry 67: 47-57.

Boosani CS, Agrawal DK (2015). Methylation and microRNA-mediated epigenetic regulation of SOCS3. Mol Biol Rep 42: 853-872.
Britton JC, Grillon C, Lissek S, Norcross MA, Szuhany KL, Chen G et al (2013). Response to learned threat: an fMRI study in adolescent and adult anxiety. Am J Psychiatry 170: 1195-1204.

Camacho A (2013). Is anxious-depression an inflammatory state? Med Hypotheses 81: 577-581.

Campos AC, Fogaca MV, Aguiar DC, Guimaraes FS (2013). Animal models of anxiety disorders and stress. Rev Bras Psiquiatr 35: S101-S111.

Chen YA, Lemire M, Choufani S, Butcher DT, Grafodatskaya D, Zanke BW et al (2013). Discovery of cross-reactive probes and polymorphic CpGs in the Illumina Infinium HumanMethylation450 microarray. Epigenetics 8: 203-209.

Chevallier N, Keller E, Maurice T (2011). Behavioural phenotyping of knockout mice for the sigma-1 (sigma(1)) chaperone protein revealed gender-related anxiety, depressive-like and memory alterations. J Psychopharmacol 25: 960-975.

Chung AS, Guan YJ, Yuan ZL, Albina JE, Chin YE (2005). Ankyrin repeat and SOCS box 3 (ASB3) mediates ubiquitination and degradation of tumor necrosis factor receptor II. Mol Cell Biol 25: 4716-4726.

Du P, Zhang X, Huang CC, Jafari N, Kibbe WA, Hou L et al (2010). Comparison of beta-value and $\mathrm{M}$-value methods for quantifying methylation levels by microarray analysis. BMC Bioinformatics 11: 587.

Du WY, Lu ZH, Ye W, Fu X, Zhou Y, Kuang CM et al (2017). The loss-of-function mutations and down-regulated expression of ASB3 gene promote the growth and metastasis of colorectal cancer cells. Chin J Cancer 36: 11.

El-Sayed AM, Haloossim MR, Galea S, Koenen KC (2012). Epigenetic modifications associated with suicide and common mood and anxiety disorders: a systematic review of the literature. Biol MoodAnxiety Disord 2: 10.

Elliott J, Johnston JA (2004). SOCS: role in inflammation, allergy and homeostasis. Trends Immunol 25: 434-440.

Erhardt A, Akula N, Schumacher J, Czamara D, Karbalai N, Muller-Myhsok B et al (2012). Replication and meta-analysis of TMEM132D gene variants in panic disorder. Transl Psychiatry 2: e156.

Erhardt A, Czibere L, Roeske D, Lucae S, Unschuld PG, Ripke S et al (2011). TMEM132D, a new candidate for anxiety phenotypes: evidence from human and mouse studies. Mol Psychiatry 16: 647-663.

Fortin JP, Labbe A, Lemire M, Zanke BW, Hudson TJ, Fertig EJ et al (2014). Functional normalization of $450 \mathrm{k}$ methylation array data improves replication in large cancer studies. Genome Biol 15: 503.

Haas BW, Canli T (2008). Emotional memory function, personality structure and psychopathology: a neural system approach to the identification of vulnerability markers. Brain Res Rev 58: 71-84.

Herder C, Bongaerts BWC, Rathmann W, Heier M, Kowall B, Koenig W et al (2013). Association of subclinical inflammation with polyneuropathy in the older population: KORA F4 Study. Diabetes Care 36: 3663-3670.

Holle R, Happich M, Lowel H, Wichmann HE, Group MKS (2005). KORA - a research platform for population based health research. Gesundheitswesen 67(Suppl 1): S19-S25.

Houseman EA, Accomando WP, Koestler DC, Christensen BC, Marsit CJ, Nelson HH et al (2012). DNA methylation arrays as surrogate measures of cell mixture distribution. BMC Bioinformatics 13: 86.

Howe AS, Buttenschon HN, Bani-Fatemi A, Maron E, Otowa T, Erhardt A et al (2016). Candidate genes in panic disorder: metaanalyses of 23 common variants in major anxiogenic pathways. Mol Psychiatry 21: 665-679.

Kendler KS, Walters EE, Neale MC, Kessler RC, Heath AC, Eaves LJ (1995). The structure of the genetic and environmental risk factors for six major psychiatric disorders in women. Phobia, generalized anxiety disorder, panic disorder, bulimia, 
major depression, and alcoholism. Archiv Gen Psychiatry 52: 374-383.

Klengel T, Binder EB (2015). Epigenetics of stress-related psychiatric disorders and gene $\mathrm{x}$ environment interactions. Neuron 86: 1343-1357.

Klengel T, Mehta D, Anacker C, Rex-Haffner M, Pruessner JC, Pariante CM et al (2013). Allele-specific FKBP5 DNA demethylation mediates gene-childhood trauma interactions. Nature Neurosci 16: 33-41.

Krebs DL, Hilton DJ (2001). SOCS proteins: negative regulators of cytokine signaling. Stem cells 19: 378-387.

Kroenke K, Spitzer RL, Williams JB, Monahan PO, Lowe B (2007). Anxiety disorders in primary care: prevalence, impairment, comorbidity, and detection. Ann Intern Med 146: 317-325.

Leussis MP, Madison JM, Petryshen TL (2012). Ankyrin 3: genetic association with bipolar disorder and relevance to disease pathophysiology. Biol Mood Anxiety Disord 2: 18.

Logue MW, Solovieff N, Leussis MP, Wolf EJ, Melista E, Baldwin C et al (2013). The ankyrin-3 gene is associated with posttraumatic stress disorder and externalizing comorbidity. Psychoneuroendocrinology 38: 2249-2257.

Marzi C, Holdt LM, Fiorito G, Tsai P-C, Kretschmer A, Wahl S et al (2016). Epigenetic signatures at AQP3 and SOCS3 engage in lowgrade inflammation across different tissues. PLoS ONE 11: e0166015.

Michopoulos V, Powers A, Gillespie CF, Ressler KJ, Jovanovic T (2017). Inflammation in fear- and anxiety-based disorders: PTSD, GAD, and beyond. Neuropsychopharmacology 42: 254-270.

Miller MK, Bang ML, Witt CC, Labeit D, Trombitas C, Watanabe K et al (2003). The muscle ankyrin repeat proteins: CARP, ankrd2/ Arpp and DARP as a family of titin filament-based stress response molecules. J Mol Biol 333: 951-964.

Montano C, Taub MA, Jaffe A, Briem E, Feinberg JI, Trygvadottir R et al (2016). Association of DNA methylation differences with schizophrenia in an epigenome-wide association study. JAMA Psychiatry 73: 506-514.

Muniz JR, Guo K, Kershaw NJ, Ayinampudi V, von Delft F, Babon JJ et al (2013). Molecular architecture of the ankyrin SOCS box family of Cul5-dependent E3 ubiquitin ligases. J Mol Biol 425: 3166-3177.

Murphy TM, O'Donovan A, Mullins N, O'Farrelly C, McCann A, Malone K (2015). Anxiety is associated with higher levels of global DNA methylation and altered expression of epigenetic and interleukin-6 genes. Psychiatr Genet 25: 71-78.

Neale BM, Kou Y, Liu L, Ma'ayan A, Samocha KE, Sabo A et al (2012). Patterns and rates of exonic de novo mutations in autism spectrum disorders. Nature 485: 242-245.

Nestler EJ, Pena CJ, Kundakovic M, Mitchell A, Akbarian S (2015). Epigenetic basis of mental illness. Neuroscientist 22: 447-463.

Pfeiffer L, Wahl S, Pilling LC, Reischl E, Sandling JK, Kunze S et al (2015). DNA methylation of lipid-related genes affects blood lipid levels. Circ Cardiovasc Genet 8: 334-342.

Segman RH, Shefi N, Goltser-Dubner T, Friedman N, Kaminski N, Shalev AY (2005). Peripheral blood mononuclear cell gene expression profiles identify emergent post-traumatic stress disorder among trauma survivors. Mol Psychiatry 10: 500-513 425.

Shimada-Sugimoto M, Otowa T, Hettema JM (2015). Genetics of anxiety disorders: genetic epidemiological and molecular studies in humans. Psychiatry Clin Neurosci 69: 388-401.
Slavich GM, Irwin MR (2014). From stress to inflammation and major depressive disorder: a social signal transduction theory of depression. Psychol Bull 140: 774-815.

Smith AK, Conneely KN, Kilaru V, Mercer KB, Weiss TE, Bradley B et al (2011). Differential immune system DNA methylation and cytokine regulation in post-traumatic stress disorder. Am J Med GenetB Neuropsychiatr Genet 156B: 700-708.

Smoller JW (2016). The genetics of stress-related disorders: PTSD, depression, and anxiety disorders. Neuropsychopharmacology 41: 297-319.

Spitzer RL, Kroenke K, Williams JB, Lowe B (2006). A brief measure for assessing generalized anxiety disorder: the GAD-7. Arch Intern Med 166: 1092-1097.

Swartz JR, Prather AA, Di Iorio CR, Bogdan R, Hariri AR (2017). A functional interleukin-18 haplotype predicts depression and anxiety through increased threat-related amygdala reactivity in women but not men. Neuropsychopharmacology 42: 419-426.

Tyrer P, Baldwin D (2006). Generalised anxiety disorder. Lancet 368: $2156-2166$.

Uddin M, Aiello AE, Wildman DE, Koenen KC, Pawelec G, de Los Santos R et al (2010). Epigenetic and immune function profiles associated with posttraumatic stress disorder. Proc Natl Acad Sci USA 107: 9470-9475.

Wang XD, Su YA, Wagner KV, Avrabos C, Scharf SH, Hartmann J et al (2013). Nectin-3 links CRHR1 signaling to stress-induced memory deficits and spine loss. Nat Neurosci 16: 706-713.

Webb BT, Guo AY, Maher BS, Zhao Z, van den Oord EJ, Kendler KS et al (2012). Meta-analyses of genome-wide linkage scans of anxiety-related phenotypes. Eur J Hum Genet 20: 1078-1084.

Wichmann HE, Gieger C, Illig TGroup MKS (2005). KORA-genresource for population genetics, controls and a broad spectrum of disease phenotypes. Gesundheitswesen 67(Suppl 1): S26-S30.

Wittchen H (1997). DIA-X-Interviews: Manual für ScreeningVerfahren und Interview Frankfurt.

Wong ML, Inserra A, Lewis MD, Mastronardi CA, Leong L, Choo J et al (2016). Inflammasome signaling affects anxiety- and depressive-like behavior and gut microbiome composition. $\mathrm{Mol}$ Psychiatry 21: 797-805.

Zannas AS, Wiechmann T, Gassen NC, Binder EB (2015). Gene-stress-epigenetic regulation of FKBP5: clinical and translational implications. Neuropsychopharmacology 41: 261-274.

Zeilinger S, Kuhnel B, Klopp N, Baurecht H, Kleinschmidt A, Gieger C et al (2013). Tobacco smoking leads to extensive genome-wide changes in DNA methylation. PLoS ONE 8: e63812.

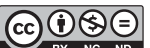

This work is licensed under a Creative Commons Attribution-NonCommercial-NoDerivs International License. The images or other third party material in this article are included in the article's Creative Commons license, unless indicated otherwise in the credit line; if the material is not included under the Creative Commons license, users will need to obtain permission from the license holder to reproduce the material. To view a copy of this license, visit http://creativecommons.org/licenses/by-nc-nd/4.0/

(c) The Author(s) 2018

Supplementary Information accompanies the paper on the Neuropsychopharmacology website (http://www.nature.com/npp) 